\title{
Impact of Alcohol Use Disorder and Agitation-related Adverse Events on Patient's Outcome in the ICU and the Following One Year after Admission
}

\author{
Vourch $\mathbf{M}^{* 1}$, Bourdiol $\mathrm{A}^{2}$, Thibault $\mathbf{M}^{3}$, Volteau $\mathrm{C}^{4}$, Zambon $\mathbf{0}^{2}$, Nicolet $\mathrm{L}^{2}$, \\ Bretonniere $\mathrm{C}^{2}$, Guitton $\mathrm{C}^{5}$ and Brule $\mathrm{N}^{2}$ \\ ${ }^{1}$ Department of Surgical Intensive Care, Nantes University Hospital, France \\ ${ }^{2}$ Medical Intensive Care Unit, Nantes University Hospital, France \\ ${ }^{3}$ Department of Emergency Medicine, Saint-Nazaire General Hospital Center, France \\ ${ }^{4}$ Biometry Platform, Research Promotion Department, Nantes University Hospital, France \\ ${ }^{5}$ Intensive Care Unit, Le Mans General Hospital Center, France
}

\section{Research Article \\ Volume 5 Issue 1}

Received Date: February 20, 2020

Published Date: February 28, 2020

DOI: $10.23880 /$ accmj- 16000170

*Corresponding author: Mickael Vourc'h, Department of Surgical Intensive Care, Hotel-Dieu, Nantes University Hospital, 44093 Nantes, France, Tel: +33685664597; Email: mickael.vourch@chu-nantes.fr

\section{Abstract}

Aims: Unhealthy alcohol use increases morbidity and agitation in the Intensive Care Units (ICU). The consequences of agitation on patients 'outcome has not been investigated so far.

Methods: Impact $\mathrm{OH}$ is a prospective observational monoester study. The objectives were to assess the incidence of agitationrelated adverse events (AE) in patients with unhealthy alcohol use and their impact on patients' outcomes.

Results: Data was collected from 159 patients, including 58\% males. Main diagnoses on admission were pneumonia (32\%) and gastro-intestinal bleeding (26\%). Eighty-seven (55\%) patients were identified as unhealthy alcohol users. AE occurred in 22/72 (31\%) low-risk drinkers vs. 36/87 (42\%) unhealthy users ( $\mathrm{p}=0.2)$. Compared with low-risk drinkers, AE was noticed in 21/40 (53\%) alcohol dependent and 16/28 (57\%) heavy alcohol users, mean differences -22, CI 95\% [-40.7; -3.2], p= 0.01 and -26 , CI 95\% [-47.8; -5.4], p= 0.02, respectively. Patients with vs. without AE spent longer mean (SD) time on ventilator [6.7 (8.3) vs. 1.5 (5.6) days, mean difference -5.2, CI 95\% [-7.7; -2.7], p< 0.0001] and had more ICU-acquired infections [16 (29\%) vs. $2(2 \%)$, mean difference -27 , CI $95 \%[-38.5 ;-14.0], \mathrm{p}<0.0001]$ without difference in mortality one year after discharge: 9 (16\%) vs. 13 (15\%), mean difference -1 , CI 95\% [-13.1; 11.5], p=0.9.

Conclusion: Alcohol dependent and heavy alcohol users presented more AE than low-risk drinkers which was associated with worse outcome, without difference in mortality one year after discharge. Hence, preventing AE in unhealthy drinkers, regardless of dependence may improve patient's outcome.

Keywords: Alcohol Use Disorder; Agitation-related adverse events, Intensive Care Unit; Alcohol Withdrawal Syndrome; Alcohol-dependence; Heavy alcohol use 
Abbreviations: AUD: Alcohol-use Disorder; ICU: Intensive Care Unit; NIAAA: National Institute on Alcohol Abuse and Alcoholism; SD: Standard Deviation; IQR: Interquartile Range.

\section{Introduction}

\section{Alcohol issue worldwide}

According to the Global Health Observatory, over 3.3 million people die from alcohol every year, representing $3 \%$ of overall mortality [1]. Alcohol is the leading psychoactive substance responsible for one death every 12 minutes in France [2]. For the National Institute on Alcohol Abuse and Alcoholism (NIAAA), alcohol consumption is unhealthy above 14 drinks a week for a men or 7 drinks a week for a women or a men above 65 years old [3]. Behind this limit patients are considered as "low-risk drinkers" (or abstinent if alcohol intake is null). At the end of the spectrum, heavy alcohol use is defined as binge drinking ( 5 drinks for men and 4 drinks for women in a period of 2 hours) occurring on 5 or more occasions in the past month. Heavy alcohol use can lead to Alcohol Use Disorder (AUD) which corresponds to excessive alcohol intake with abuse or dependence [4].

\section{Alcohol in the ICU}

In the Intensive Care Unit (ICU) AUD ranges from 10 to $33 \%$ Moss and Burnham [5] and was reported to increase the risk of infection [6], hospital length of stay, time on ventilator and mortality [7]. Nevertheless, Gacouin, et al. [8] reported that compared to low-risk, unhealthy alcohol users had a higher risk of death and spent longer time on ventilator, regardless of AUD. These results suggest that even for moderate intake, regular alcohol use leads to specific issues which can impact patient's outcome in the ICU. Indeed, chronic alcohol exposure (namely GABA agonist) leads to the downregulation of the GABA-A receptor and the upregulation of the NMDA receptors [9] which can lead to sympathetic manifestations and/or psychomotor agitation upon discontinuation. Interestingly, agitation is frequent in alcohol users [10]. Whether agitation and its related adverse events are possible explanations for the altered outcome in the ICU is unknown.

This pilot study was built to assess the incidence of agitation-related adverse events (further referred as AE) in the ICU according to the alcohol intake and their impacts on patients' outcome.

\section{Methods}

\section{Study design,Settings andEthicalConsiderations}

IMPACT-OH study is a prospective single center observational study. All consecutive patients admitted to the medical ICU of Nantes University Hospital from June to September 2012, were included and followed over a one year period until September 2013. Minors or adults under protection were not included. Written and oral information were delivered to the patient and/or his nextof-kin on admission. The protocol was approved by the Ethics Committee of the Society de Reanimation de Langue Françoise (CE SRLF 12-389).

\section{Data Collection and Endpoints}

On admission, the diagnosis, medical history, clinical, biological and demographic data were collected for all patients. Alcohol intake, CAGE score [11] and Smast-Test were used to classified patients as follows: Low-risk drinkers (including abstinent), unhealthy and heavy alcohol users were defined according to the NIAAA threshold as described in section 1.1 [3]. Alcohol dependence was considered for a CAGE score of 2 or more or a Smast-Test score [12] of 3 or more. Alcohol abuse and AUD were diagnosed as previously described [13]. When patients died or were unable to answer the specific questionnaires for alcohol intake, data was recorded on behalf of their next-of-kin. The one year follow up was assessed by phone call to the patient, family or caregivers for severely disabled patients.

The primary objective was to determine the incidence of AE in patients with alcohol intake above the NIAAA threshold. The latter were defined as unplanned extubation, medical disposal removal, immobilization device or heavy sedation requirement. The secondary objective was to determine the consequences of $\mathrm{AE}$ on patients' outcome in the ICU: time on ventilator; ICU-acquired infection; hospital-acquired pneumonia; extubation failure; discharge in a psychiatric unit and mortality one year after discharge.

\section{Standard of Care in Sedation and Analgesia}

For patients under mechanical ventilation the level of sedation and analgesia were adjusted to obtain a score equal to zero on the Richmond Agitation-Sedation Scale [14] and inferior to 5 on the Behavior pain scale [15]. In case of agitation continuous intravenous infusions of fentanyl from 0.02 to 2 micrograms $(\mu \mathrm{g})$ per kilogram $(\mathrm{kg})$ per hour (h) and midazolam from 0.05 to 0.5 milligrams (mg) $/ \mathrm{kg} / \mathrm{h}$ were initiated. Heavy sedation corresponded to a continuous infusion of midazolam superior to $0.5 \mathrm{mg} / \mathrm{kg} / \mathrm{h}$ to obtain a score lower than 1 on the Richmond Agitation-Sedation Scale. Patients with AUD who did not require mechanical ventilation were monitored using the Cushman score [16] to detect alcohol withdrawal syndrome every four hours. A standard oral dose of Diazepam $10 \mathrm{mg}$ was administered every two hours for Cushman score between 4 and 6 . If the 
score was higher than 7, an injectable dose of Diazepam 10 mg was administered and repeated every hour to obtain a score below 7 .

ICU-acquired Infections were defined as follows: Hospital-acquired pneumoniae which occurred after the first 48 hours of hospitalization were considered as nosocomial pneumoniae according to the American Thoracic Society guidelines [17] Catheter-related bloodstream infections were confirmed by paired blood samples drawn from both the suspected catheter and a peripheral vein [18] Only complicated urinary tract infections were recorded (pyelonephritis or any urinary tract infection associated with bacteremia or sepsis).

\section{Data Sharing Statements}

De-identified data collected for the study, including individual participant data and a data dictionary defining each field in the set will be made available to others. Statistical analysis plan, informed consent form and full dataset will be made available on reasonable request by addressing an email to the corresponding author within five years following publication.

\section{Statistical Analysis}

Baseline characteristics were described for each group. Continuous variables were presented with mean and standard deviation (SD), or median and interquartile range (IQR) when the assumption of normality was not met. Categorical data were expressed as numbers and percentages. The outcomes were compared between the groups using the Chi-
2 or Fischer tests for qualitative outcomes and Student test for quantitative outcomes. Statistical tests were two-sided with a statistical significance of $\mathrm{p}<0.05$. SAS software $v 9.3 \AA$ was used for all statistical analyses.

\section{Results}

Over a four-month period, 212 patients were admitted in ICU. Four patients met exclusion criteria and dataset was incomplete in 49 patients. Finally, full dataset was available for 159 patients.

\section{Patients Characteristics}

Baseline characteristics of the population are shown in Table 1. According to the NIAAA classification, 72 patients (45\%) were classified as low-risk and 87 (55\%) as unhealthy alcohol users [3]. AUD was identified in 71/87 (82\%) patients 40 (56\%) with dependence. Heavy alcohol use was found in 28/71 (40\%) patients including 2 patients without AUD (Figure 1). Main diagnoses at admission were pneumonia (32\%), gastro-intestinal bleeding (26\%), multiple trauma $(12 \%)$ or intoxication $(12 \%)$ without difference between low-risk and unhealthy users $(p=0.1)$. Mean SAPS II score was $38.5(+/-34.1)$ in unhealthy alcohol users vs. $39.3(+/-$ $16.5)$ in low-risk $(p=0.9)$. Admission in the ICU was more frequently related to acute alcohol or chronic alcohol intake $(p=0.0003)$ in unhealthy users, and their medical history included more polyneuritis ( $15 \%$ vs. $4 \%, p=0.02)$, seizures ( $9 \%$ vs. $1 \%, p=0.04)$, accidental traumas $(17 \%$ vs $3 \%, p=$ $0.03)$, injuries following a fight ( $15 \%$ vs $0 \%, p=0.0006)$ or depressions $(19 \%$ vs $3 \%, p=0.002)$ than low-risk patients.

\begin{tabular}{|c|c|c|c|c|c|}
\hline & $\begin{array}{c}\text { Whole population } \\
\mathbf{N = 1 5 9}\end{array}$ & $\begin{array}{c}\text { Low-risk drinkers } \\
\mathbf{N = 7 2}(\mathbf{4 5} \mathbf{0})\end{array}$ & $\begin{array}{c}\text { Unhealthy uses } \\
\mathbf{N = \mathbf { 8 7 } ( 5 5 \% )}\end{array}$ & $\boldsymbol{p}$ & $\begin{array}{c}\text { Difference CI } \\
\mathbf{9 5} \%\end{array}$ \\
\hline Age, mean (SD), year & $54(18.3)$ & $56(17.1)$ & $52(18.3)$ & 0.2 & $4[-1.8 ; 9.4]$ \\
\hline Male, n (\%) & $92(58)$ & $34(47)$ & $58(67)$ & 0.01 & $-20[-34.7 ; 4.2]$ \\
\hline Knaus Score, n (\%) & & & & 0.6 & - \\
\hline A & $89(56)$ & $40(56)$ & $49(56)$ & & \\
\hline B & $46(29)$ & $20(28)$ & $26(30)$ & & \\
\hline C & $22(14)$ & $12(17)$ & $10(12)$ & & \\
\hline D & $2(1)$ & $0(0)$ & $2(2)$ & & \\
\hline Diagnosis at admission, n (\%) & & & & 0.1 & \\
\hline Pneumonia & $51(32)$ & $27(37)$ & $24(28)$ & & \\
\hline Gastro intestinal bleeding & $42(26)$ & $18(25)$ & $24(28)$ & & \\
\hline Multiple trauma & $19(12)$ & $18(25)$ & $1(1)$ & & \\
\hline Intoxication & $19(12)$ & $6(9)$ & $13(4)$ & & \\
\hline Other & $28(18)$ & $3(4)$ & $25(29)$ & & \\
\hline
\end{tabular}


Anaesthesia \& Critical Care Medicine Journal

\begin{tabular}{|c|c|c|c|c|c|}
\hline ICU admission, $\mathrm{n}(\%)$ & & & & 0.0003 & - \\
\hline Related to acute intake & $17(11)$ & $4(5)$ & $13(15)$ & & \\
\hline Related to chronic intake & $21(13)$ & $0(0)$ & $21(24)$ & & \\
\hline \multicolumn{6}{|l|}{ Medical history, n (\%) } \\
\hline Cirrhosis & $8(5)$ & $3(4)$ & $5(6)$ & 0.7 & $-2[-8.5 ; 5.2]$ \\
\hline Polyneuritis & $16(10)$ & $3(4)$ & $13(15)$ & 0.02 & $-11[-20.0 ;-2.1]$ \\
\hline Seizure & $9(6)$ & $1(1)$ & $8(9)$ & 0.04 & $-8[-14.8 ;-1.2]$ \\
\hline Withdrawal syndrome & $11(7)$ & $0(0)$ & $11(12)$ & 0.01 & $-11[-17.7 ; 3.0]$ \\
\hline Accidental trauma & $17(11)$ & $2(3)$ & $15(17)$ & 0.03 & $-14[-23.5 ; 5.7]$ \\
\hline Injury following a fight & $13(8)$ & $0(0)$ & $13(15)$ & 0.0006 & $-15[-23.0 ; 7.6]$ \\
\hline Depression & $18(11)$ & $2(3)$ & $16(19)$ & 0.002 & $-16[-25.2 ; 6.9]$ \\
\hline Macrocytosis & $20(13)$ & $0(0)$ & $20(24)$ & 0.0001 & $-24[-32.6 ; 14.5]$ \\
\hline Thrombopaenia & $6(4)$ & $0(0)$ & $6(7)$ & 0.03 & $-7[-12.5 ;-1.6]$ \\
\hline \multicolumn{6}{|l|}{ Other addiction, n (\%) } \\
\hline Tabacco & $62(39)$ & $22(31)$ & $40(47)$ & 0.04 & $-16[-31.0 ; 1.0]$ \\
\hline Cannabis & $9(6)$ & $0(0)$ & $9(11)$ & 0.004 & $-11[-17.5 ;-4.2]$ \\
\hline Other narcotic drugs & $0(0)$ & $0(0)$ & $4(5)$ & 0.1 & $-5[-9.4 ;-0.2]$ \\
\hline SAPS II, mean (SD) ${ }^{\mathrm{b}}$ & $38.8(18.8)$ & $39.3(16.5)$ & $38.5(34.1)$ & 0.9 & $0.8[-5.1 ; 6.8]$ \\
\hline SOFA score, mean (SD) ${ }^{c}$ & $5.3(3.6)$ & $5.3(3.8)$ & $5.5(3.3)$ & 0.9 & $-0.2[-1.1 ; 1.1]$ \\
\hline
\end{tabular}

${ }^{a}$ Chronic health status: Class A, normal health status; Class B, moderate activity limitation; Class C, severe activity limitation due to chronic disease; Class D, bedridden patient.

Table 1: Baseline characteristics of low-risk and unhealthy alcohol use.

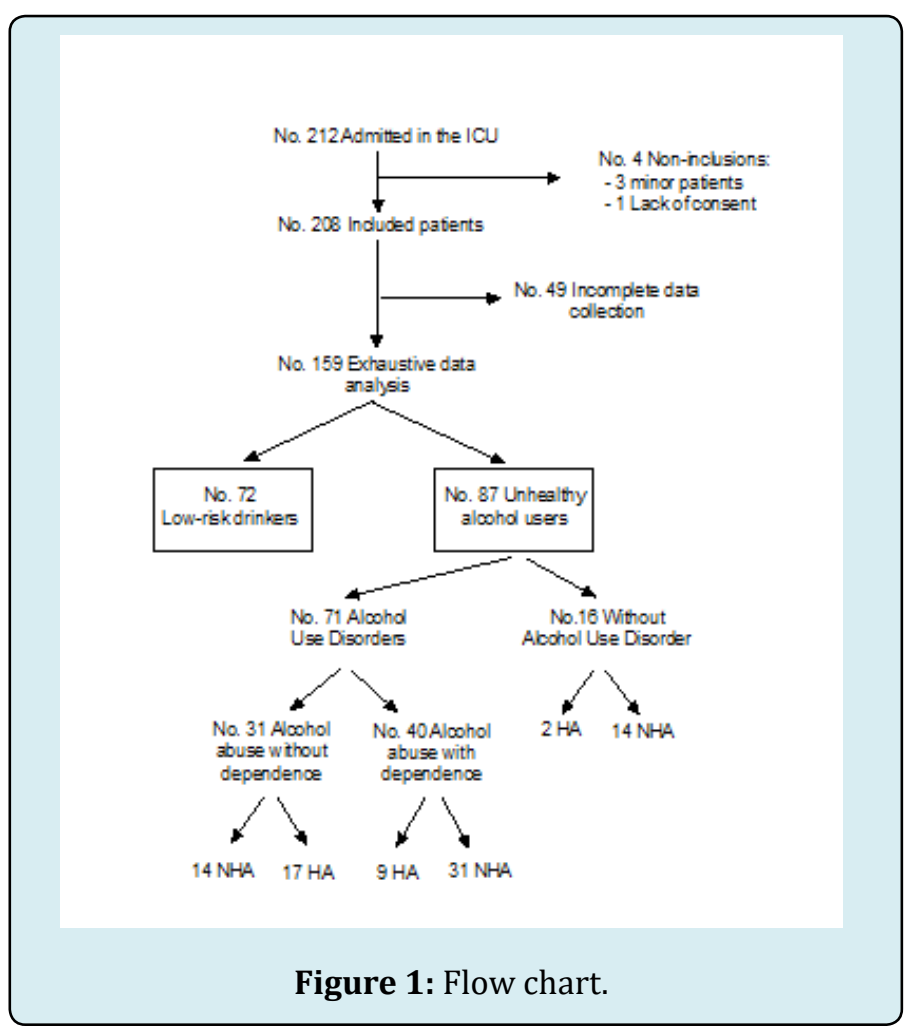

Figure 1 on admission, patients was classified as lowrisk drinkers or unhealthy alcohol users depending on their weekly alcohol intake, respectively below or above the NIAAA threshold. Alcohol use disorder (AUD) includes alcohol abuse with or without alcohol dependence. HA: Heavy alcohol users, NHA: Non-heavy alcohol users.

\section{Incidence of agitation-related adverse events}

Agitation is a challenging issue in the ICU because it can compromise medical care [19]. Patients were classified according to their alcohol consumption (Figure 1) and the incidence of $\mathrm{AE}$ was assessed for each class (Table 2). It occurred more frequently in unhealthy alcohol users compared with low-risk drinkers, respectively 36 (42\%) vs. $22(31 \%)$, without significant difference $(p=0.2)$. Because alcohol dependent and heavy alcohol users showed the highest rate of AE (Table 3) compared with low-risk drinkers, these 3 groups were compared. Alcohol-dependent and heavy users showed a significantly higher rate of $\mathrm{AE}$ than low-risk drinkers, respectively 21 (53\%) vs. 22 (31\%), mean difference -22, CI 95\% [-40.7; -3.2], $p=0.02$ and 16 (57\%) vs. 22 (31\%), mean difference -26 , CI 95\% [-47.8; -5.4], $p=$ 0.01 . When each adverse event were taken separately, heavy alcohol use was associated with more heavy sedation than 


\begin{tabular}{|c|c|}
\hline $\mathbf{N}=\mathbf{5 8} / \mathbf{1 5 9}(\mathbf{3 6} \%)$ & No. patients with agitation-related adverse events \\
\hline Low-risk users & $22 / 72(31 \%)$ \\
\hline Unhealthy alcohol users & $36 / 87(42 \%)$ \\
\hline Without AUD & $6 / 16(38 \%)$ \\
\hline Heavy alcohol use & $2 / 2(100 \%)$ \\
\hline No Heavy alcohol use & $4 / 14(29 \%)$ \\
\hline With AUD & $30 / 71(42 \%)$ \\
\hline With dependence & $21 / 40(53 \%)$ \\
\hline Heavy alcohol use & $7 / 9(78 \%)$ \\
\hline No Heavy alcohol use & $14 / 31(45 \%)$ \\
\hline Without dependence & $9 / 31(29 \%)$ \\
\hline Heavy alcohol use & $7 / 17(41 \%)$ \\
\hline No Heavy alcohol use & $2 / 14(14 \%)$ \\
\hline
\end{tabular}

Incidence of agitation-related adverse events according to alcohol consumption.

Table 2: The occurrence of AE was measured for each class of alcohol users: Low-risk (or abstinent); Unhealthy alcohol users (respectively under or above the threshold of NIAAA alcohol intake); Patients with heavy alcohol use, alcohol use disorder (AUD), with or without dependence.

\begin{tabular}{|c|c|c|c|c|c|c|c|}
\hline & $\begin{array}{c}\text { Low-risk } \\
\text { drinkers } \\
\mathbf{N = 7 2}\end{array}$ & $\begin{array}{c}\text { Alcohol- } \\
\text { dependent N } \\
\mathbf{9 4 0}\end{array}$ & $\boldsymbol{p}^{*}$ & $\begin{array}{c}\text { Difference CI } \\
\mathbf{9 5 \%}\end{array}$ & $\begin{array}{c}\text { Heavy } \\
\text { alcohol } \\
\text { users N = 28 }\end{array}$ & $\boldsymbol{p}^{*}$ & $\begin{array}{c}\text { Difference CI } \\
\mathbf{9 5 \%}\end{array}$ \\
\hline $\begin{array}{c}\text { At least 1 agitation-related } \\
\text { adverse event in ICU, n (\%) }\end{array}$ & $22(31)$ & $21(53)$ & 0.02 & $-22[-40.7 ;-3.2]$ & $16(57)$ & 0.01 & $-26[-47.8 ;-5.4]$ \\
\hline Unplanned extubation & $3(4)$ & $3(8)$ & 0.7 & $-4[-6.1 ; 12.7]$ & $2(7)$ & 0.6 & $-3[-7.7 ; 13.5]$ \\
\hline Medical disposal removal & $3(4)$ & $2(5)$ & 1 & $-1[-7.4 ; 9.0]$ & $2(7)$ & 0.6 & $-3(-7.6 ; 13.6]$ \\
\hline $\begin{array}{c}\text { Immobilization device } \\
\text { removal }\end{array}$ & $10(14)$ & $9(23)$ & 0.2 & $-9[-23.8 ; 6.6]$ & $8(29)$ & 0.09 & $-15[-33.2 ; 3.9]$ \\
\hline Heavy sedation & $19(26)$ & $16(40)$ & 0.1 & $-14[-31.9 ; 4.7]$ & $14(50)$ & 0.02 & $-24[-44.7 ;-2.5]$ \\
\hline Seizure, n (\%) & $1(1)$ & $5(13)$ & 0.02 & $-12[-22.2 ;-0.6]$ & $4(14)$ & 0.02 & $-13[-26.1 ;-0.1]$ \\
\hline
\end{tabular}

l Agitation-related adverse events and seizure in the ICU.

*P-value compared to low-risk drinkers

${ }^{\text {aS }}$ ome patients presented one or more agitation-related adverse events.

bHeavy sedation corresponded to a continuous infusion of Midazolam superior to $0.5 \mathrm{mg} / \mathrm{kg} / \mathrm{h}$ to obtain a Richmond Agitation Sedation Scale lower than +1 .

Table 3: Heavy alcohol users included 26 patients with Alcohol Use Disorder (AUD) [9 with and 17 without dependence] and 2 patients without AUD.

\section{Agitation-Related Adverse Events and Outcome}

Considering the reduced size of the sample, the impact of $\mathrm{AE}$ on the secondary outcomes was assessed in all the patients regardless of their alcohol intake. In this sample, 10 (14\%) low-risk drinkers and $8(9 \%)$ unhealthy users died in the ICU over the study period $(p=0.9)$. Since death was likely to interfere with the occurrence of $\mathrm{AE}$, these patients were excluded from this analysis (Table 4). In patients with at least one $\mathrm{AE}$, time on ventilator was longer and the rate of ICU-acquired infections was higher vs. patients without AE, respectively: 6.7 (8.3) vs. 1.5 (5.6) days, mean difference -5.2 , CI 95\% [-7.7; -2.7$], p<0.0001)$ and $16(29 \%)$ vs. $2(2 \%)$, mean difference -27 , CI 95\% [-38.5; -14.0], $p<0.0001)$. Finally, discharge from the ICU in a psychiatric unit was more frequent in patients with vs. without $\mathrm{AE}(14 \%$ vs. $4 \%$, 


\section{Anaesthesia \& Critical Care Medicine Journal}

mean difference -10 , CI 95\% [-16.7; -2.7], $p=0.01)$, without difference in mortality one year after discharge: $9(16 \%)$ vs.
13 (15\%), mean difference -1 , CI 95\% [-13.1; 11.5], $p=0.9$.

\begin{tabular}{|c|c|c|c|c|}
\hline & $\begin{array}{c}\text { Noagitation- } \\
\text { related adverse } \\
\text { event N = 85 }\end{array}$ & $\begin{array}{c}\text { Agitation-related } \\
\text { adverse event N } \\
\mathbf{=} \mathbf{5 6}\end{array}$ & $\boldsymbol{p}$ & Difference CI 95\% \\
\hline Time on ventilator, mean (SD), days & $1.5(5.6)$ & $6.7(8.3)$ & $<0.0001$ & $-5.2[-7.7 ;-2.7]$ \\
\hline Extubation failure, $\mathrm{n}(\%)^{\mathrm{a}}$ & $0(0)$ & $3(5)$ & 0.06 & $-5[-11.3 ; 0.54]$ \\
\hline ICU-acquired infection, $\mathrm{n}(\%)^{\mathrm{b}}$ & $2(2)$ & $16(29)$ & $<0.0001$ & $-27[-38.5 ;-14.0]$ \\
\hline${\text { Hospital-acquired pneumoniae, } \mathrm{n}(\%)^{\mathrm{c}}}^{\mathrm{b}}$ & $0(0)$ & $11(20)$ & $<0.0001$ & $-20[-30.1 ;-9.2]$ \\
\hline Discharge from ICU in a psychiatric unit, $\mathrm{n}(\%)$ & $3(4)$ & $8(14)$ & 0.04 & $-10[-16.7 ;-2.7]$ \\
\hline Mortality 1 year after discharge, $\mathrm{n}(\%)$ & $13(15)$ & $9(16)$ & 0.9 & $-1[-13.1 ; 11.5]$ \\
\hline
\end{tabular}

Impact of agitation-related adverse events on patients' outcomes.

${ }^{a}$ Extubation failure was defined as the need to reinsulate within 72 hours of extubation [26].

bICU-acquired infection encompassed hospital-acquired pneumonia, confirmed catheter-related bloodstream infections and complicated urinary tract infections associated with sepsis or bacteremia.

'Hospital-acquired pneumonia was defined as pneumonia occurring after the first 48 hours of hospitalization.

Table 4: This analysis excluded patients who died in the ICU (i.e. 18 patients: 16 patients without agitation-related adverse event (AE) and 2 patients with $\mathrm{AE}$ ).

\section{Discussion}

\section{Main Results}

The IMPACT-OH study highlighted that firstly, AE is frequent in the ICU ( $36 \%$ of the whole population), especially in unhealthy alcohol users (42\%), alcohol-dependent (53\%) and heavy alcohol users (57\%). Secondly, AE was associated with worse clinical outcome including longer time on ventilator $(p<0.0001)$ and ICU-acquired infection $(p<0.0001)$ and occurred regardless of alcohol dependence (i.e. 2 heavy alcohol users did not have AUD criterium). As a result, $\mathrm{AE}$ stands as one possible explanation of the increased morbidity in unhealthy alcohol users described previously in the ICU Gacouin, et al. [8]. This strengthens the idea that systematic assessment of alcohol intake could prove beneficial to foresee the risk of $\mathrm{AE}$ and set preventive strategies. Despite the standardization of sedation based on RASS and BPS scale, $36 \%$ of the whole population and $42 \%$ of unhealthy users showed $\mathrm{AE}$, driving extensive research in this field. Indeed, the amount of sedation to prevent agitation is difficult to adjust given the inter-individual variation for drug susceptibility.

Agitation remains a major issue in the ICU and was reported to increase morbidity [20], cognitive impairment [21] and hospital costs [22]. In the present monocentric study, the prevalence of unhealthy alcohol users was higher than previously described [8]. The latter study included patients staying 3 days or more in the ICU whereas IMPACT$\mathrm{OH}$ study included all consecutive patients regardless of their length of stay. These discrepancies and the smaller sample size may also explain the lack of difference regarding one year mortality rate compared with Gacouin, et al. study [8]. At the opposite, Stewart et al. reported a higher incidence of agitation in unhealthy alcohol users (55\%) defined by the Bloomsbury Sedation scale score [10]. Regardless of alcohol dependence, chronic alcohol intake leads to imbalance the activation of GABA vs. NMDA pathways which favors psychomotor agitation upon alcohol discontinuation. This may explain the high rate of agitation in unhealthy drinkers regardless of AUD. In the same way, the significant increase of discharge in psychiatric units is in line with previous reports suggesting that agitation could give rise to mid- or long-term cognitive impairment [21].

\section{Limitations}

Several limits of the study should be discussed. When analyzing the impact of AE on patients' outcome: 1) The increased time on ventilator in patients with $\mathrm{AE}$ have to be tempered because this parameter can also favor agitation by it-self: 2) The exclusion of patients who died in the ICU is a possible bias of the analysis. Consequently, the impact of $\mathrm{AE}$ on mortality in the ICU was not assessed. The rate of AE between low-risk (31\%) vs. unhealthy alcohol users (42\%) was not statistically different. However, the small size of the sample and the frequent self- or next-of-kin under-reporting of the actual alcohol consumption may explain this result. The incidence of alcohol withdrawal syndrome (i.e. alcohol dependent patients) was not assessed during agitation. However, the study focused on the incidence of agitation regardless of dependence. The definition of $\mathrm{AE}$ used in the present study was not reported previously but matched with 


\section{Anaesthesia \& Critical Care Medicine Journal}

clinically relevant issues. Moreover, it allowed characterizing patients with longer time on ventilator, and increased risk of ICU-acquired infection. Nevertheless, the design of the study did not allow to establish causality between pejorative outcomes and agitation.

Up to now, the prevention of agitation in unhealthy alcohol users in the ICU has not been studied in large randomized trial: In these settings, benzodiazepines stand as an independent risk factor of delirium in ICU; dexmedetomidine raised controversial results [23] the administration of baclofen was reported to be feasible [24] and may reduce both agitation and the use of benzodiazepines [25]. These results urged us to build a large randomized study to assess baclofen vs. placebo to prevent agitation in unhealthy alcohol users [26].

\section{Conclusion}

The present study highlighted the high incidence of agitation-related adverse events in the ICU, especially among unhealthy alcohol users, and their consequences on shortterm outcome. These results underline the shortcomings of our current strategies, warranting a thorough assessment of alcohol consumption upon ICU admission and further studies to better prevent agitation.

\section{References}

1. Gulland A (2014) Alcohol consumption kills 3.3 million people a year, WHO calculates. BMJ 348: g3335.

2. Guerin S, Laplanche A, Dunant A, Hill C (2013) Alcoholattributable mortality in France. Euro J Pub Heal 23(4): 588-593.

3. Saitz, R (2005) Clinical practice. Unhealthy alcohol use. N Engl J m 352(6): 596-607.

4. Schuckit MA (2009) Alcohol-use disorders. Lancet 373(9662): 492-501.

5. Moss M, Burnham EL (2006) Alcohol abuse in the critically ill patient. Lancet 368(9554): 2231-2242.

6. Gacouin A, Legay F, Camus C, Volatron AC, Barbarot N, et al. (2008) At-risk drinkers are at higher risk to acquire a bacterial infection during an intensive care unit stay than abstinent or moderate drinkers. Crit Care Med 36(6): 1735-1741.

7. OBrien JM, Lu B, Ali NA, Martin GS, Aberegg SK, et al. (2007) Alcohol dependence is independently associated with sepsis, septic shock, and hospital mortality among adult intensive care unit patients. Crit Care Med 35(2): 345-350.
8. Gacouin A, Tadie JM, Uhel F, Sauvadet E, Fillâtre P, et al. (2014) At-risk drinking is independently associated with ICU and one-year mortality in critically ill nontrauma patients. Crit Care Med 42(4): 860-867.

9. Carlson RW, Kumar NN, Wong-Mckinstry E, Ayyagari, Puri N, et al. (2012) Alcohol withdrawal syndrome. Crit Care Clin 28(4): 549-585.

10. Stewart D, Kinsella J, McPeake J, Quasim T, Puxty A (2018) The influence of alcohol abuse on agitation, delirium and sedative requirements of patients admitted to a general intensive care unit. J Intensive Care Soc 20(3): 208-215.

11. Ewing JA (1984) Detecting alcoholism. The CAGE questionnaire. JAMA 252(14): 1905-1907.

12. Selzer ML, Vinokur A, van Rooijen L (1975) A selfadministered Short Michigan Alcoholism Screening Test (SMAST). J Stud Alcohol 36(1): 117-126.

13. Tyburski EM, Sokolowski A, Samochowiec J, Samochowiec A (2014) New diagnostic criteria for alcohol use disorders and novel treatment approaches 2014 update. Arch Med Sci 10(6): 1191-1197.

14. Sessler CN, Gosnell MS, Grap MJ, Brophy GM, ONeal PV, et al. (2002) The Richmond Agitation-Sedation Scale: validity and reliability in adult intensive care unit patients. Am J Respir Crit Care Med 166(10): 1338-1344.

15. Payen JF, Bru O, Bosson JL, Lagrasta A, Novel E, et al. (2001) Assessing pain in critically ill sedated patients by using a behavioral pain scale. Crit Care Med 29(12): 2258-2263.

16. Cushman PP, Forbes RR, Lerner WW, Stewart MM (1985) Alcohol withdrawal syndromes: clinical management with lofexidine. Alcohol Clin Exp Res 9(2): 103-108.

17. American Thoracic Society, Infectious Diseases Society of America (2005) Guidelines for the management of adults with hospital-acquired, ventilator-associated, and healthcare-associated pneumonia. Am J Respir Crit Care Med 171(4): 388-416.

18. Mermel LA, Allon M, Bouza E, Craven DE, Flynn P, et al. (2009) Clinical practice guidelines for the diagnosis and management of intravascular catheter-related infection: 2009 Update by the Infectious Diseases Society of America. Clin Infect Dis 49(1): 1-45.

19. Siegel MD (2003) Management of agitation in the intensive care unit. Clin Chest Med 24(4): 713-725.

20. Ely EW, Shintani A, Truman B, Speroff T, Gordon SM, et al. (2004) Delirium as a predictor of mortality in 


\section{Anaesthesia \& Critical Care Medicine Journal}

mechanically ventilated patients in the intensive care unit. JAMA 291(14): 1753-1762.

21. Girard TD, Jackson JC, Pandharipande PP, Pun BT, Thompson JL, et al. (2010) Delirium as a predictor of long-term cognitive impairment in survivors of critical illness. Crit Care Med 38(7): 1513-1520.

22. Milbrandt EB, Deppen S, Harrison PL, Shintani AK, Speroff T, et al. (2004) Costs associated with delirium in mechanically ventilated patients. Crit Care Med 32(4): 955-962.

23. Mueller SW, Preslaski CR, Kiser TH, Fish DN, Lavelle JC, et al. (2014) A randomized, double-blind, placebocontrolled dose range study of dexmedetomidine as adjunctive therapy for alcohol withdrawal. Crit Care Med
42(5): 1131-1139.

24. Vourch M, Dailly E, Hourmant Y, Bellouard R, Mahe PJ, et al. (2019) Pharmacokinetics and toxicity of high-dose baclofen in ICU patients. Prog Neuropsychopharmacol Biol Psychiatry 92: 450-456.

25. Addolorato G, Leggio L, Abenavoli L, Agabio R, Caputo $F$, et al. (2006) Baclofen in the treatment of alcohol withdrawal syndrome: a comparative study vs diazepam. Am J med 119(3): 276.

26. Vourch M, Feuillet F, Mahe PJ, Sebille V, Asehnoune K, et al. (2016) Baclofen to prevent agitation in alcohol-addicted patients in the ICU: study protocol for a randomised controlled trial. Trials 17(1) 415. 